\title{
THE DEPOLARIZATION PROPERTIES OF CIRRUS CLOUD BY LIDAR: OBSERVATION AND MODEL MATCH
}

\author{
Zhenzhu Wang ${ }^{*}, 1$, Anatoli Borovoi ${ }^{2}$, Gennady Matvienko ${ }^{2}$, Dong Liu ${ }^{1}$, Chenbo Xie ${ }^{1}$, Yingjian \\ Wang $^{1,3}$, Alexander Konoshonkin ${ }^{2}$, Natalia Kustova ${ }^{2}$ \\ ${ }^{1}$ Key Laboratory of Atmospheric optics, Anhui Institute of Optics and Fine Mechanics, Chinese Academy \\ of Sciences, Hefei, Anhui 230031, China \\ ${ }^{2} V$. E. Zuev Institute of Atmospheric Optics, Rus. Acad. Sci, Tomsk, 634055, Russia \\ ${ }^{3}$ University of Science and Technology of China, Hefei, Anhui 230031, China \\ *Email: zzwang@aiofm.ac.cn
}

\begin{abstract}
The Polarization Lidar has been developed for cirrus cloud optical properties measurement. The Nd-YAG laser is employed as emitting linear source and two channels are used for detecting back-scattering signals from cirrus cloud including two $532 \mathrm{~nm}$ Orthogonal Polarization channels. Measurements were carried out from December 2010, to February 2013. In this work, we consider the only linear depolarization ratio measured at a wavelength of $532 \mathrm{~nm}$. The dependences of depolarization ratios on both the size of ice crystals and the distribution of their distortion angles were also calculated. These results were compared with each other and used for retrieving the microphysical parameters of cirrus clouds observed in Hefei.
\end{abstract}

\section{INTRODUCTION}

Cirrus cloud is one of the most commonly occurring cloud types globally. It forms in the upper part of troposphere, due to synoptic scale lifting, or as a result of moisture transport by deep convection [1-3]. Cirrus clouds cover about 30\% of the Earth surface and they essentially impact on the radiative budget of the Earth and, consequently, on the climate [4-5]. The radiative characteristics of cirrus clouds are poorly studied yet because of the strong spatial and temporal variability of the clouds. And it is also a significant challenge to accurately measure and model their optical and microphysical properties in order to assimilate them into global climate models. On the other hand, lidar has been extensively demonstrated for observing cloud properties. The previous work with lidar techniques concentrate on cirrus clouds in experiment [6-12] and theory [13-14].

\section{METHODOLOGY}

\subsection{The lidar system}

The Three-wavelength Raman Mie Polarization Lidar (named as TRMPL) has been developed for cirrus cloud properties measurement. This idar system has built in Hefei and passed the performance assessment in the end of 2010, and then improved to be a movable lidar system to carry out the continuous measurements of vertical distribution of regional cloud.

The TRMPL is a moveable and continuous working lidar installed in a standard container with a glass window on the roof. Fig. 1 gave the system diagram and the photo of the TRMPL.For this paper, only polarization signals were used for cirrus clouds.

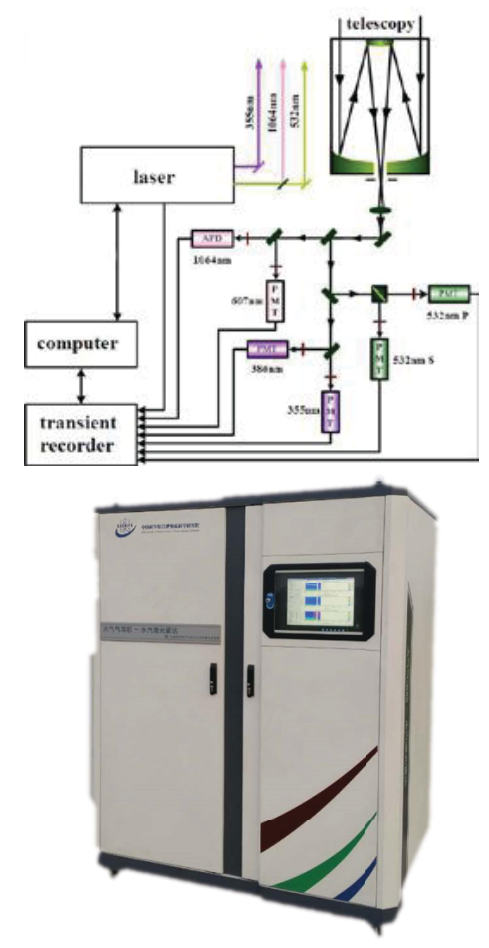

Figure 1. The diagram and photo of TRMPL

\subsection{The inversion methods}


In general, the depolarization ratio (namely) is determined from parallel $\left(\mathrm{P}_{\mathrm{p}}\right)$ and perpendicular $\left(\mathrm{P}_{\mathrm{s}}\right)$ polarization signals at $532 \mathrm{~nm}$ together with the $\mathrm{k}$ value $[7-8]$ :

$$
\delta=\sigma_{\perp} / \sigma_{/ /}=\kappa \cdot \mathrm{P}_{\mathrm{s}} / \mathrm{P}_{\mathrm{p}}
$$

The theoretical problem of light backscattering by ice crystals of cirrus clouds has been solved only for the simplest crystal shapes-hexagonal columns and plates of an ideal shape [15]. In this work, we present the results of calculations for a more realistic model, that is, randomly oriented distorted hexagonal columns, where hexagonal faces of a crystal are skewed relative to rectangular faces at a small angle $\xi$ (distortion angle), similarly to [16]. At such a distortion of the crystal shape, the right angle between crystal faces results in the strong dependence of the backscattering on the crystal sizes.

However, averaging over the distortion angles $\xi$, inherent in real clouds, levels the dependence of the depolarization ratio on the crystal sizes. Thus, as our calculations show, the measured values of the depolarization ratio can be meant to weakly depend on crystal sizes. Consequently, the depolarization ratio can be considered as an indicator of only the crystal shape and its spatial orientation in a cloud.

\section{RESULTS}

The statistical characteristics of depolarization ratio for cirrus cloud have been investigated using measured data from Dec. of 2010 to Feb. of 2013, shown in figure 2 . The averaged depolarization ratio is 0.36 at $532 \mathrm{~nm}$ varying almost from 0.2 to 0.5 . In this work, we calculate the depolarization ratio as a function of crystal sizes at different distributions of crystal distortion angles for the given model of crystal shape. This allows us to retrieve, with a known uncertainty, microphysical parameters of cirrus clouds, observed at Heifei in this period.

Sizes of hexagonal columns are defined by one parameter L (the column length). The diameter D of a hexagonal column in this case is found from the empirical relation [17]. Figure 3 shows depolarization ratios calculated in the physical optics approximation for randomly oriented hexagonal columns, with the distortion angle $\xi$ distributed by the Gauss law. As seen in Figure 3, the sufficiently strong distortion of crystal shape $\left(\xi\right.$ eff $>3^{\circ}$ ) levels the dependence of the depolarization ratio on the crystal sizes and increases it from $\delta \approx 0.2$, which is typical for crystals of regular shape, to $\delta \approx 0.35$.

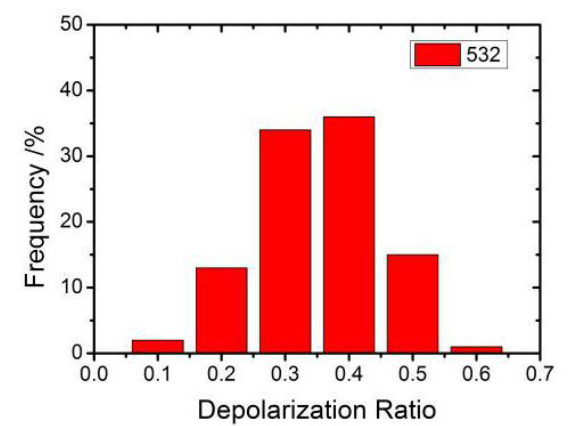

Figure 2. The frequency of depolarization ratio for cirrus observed at Heifei

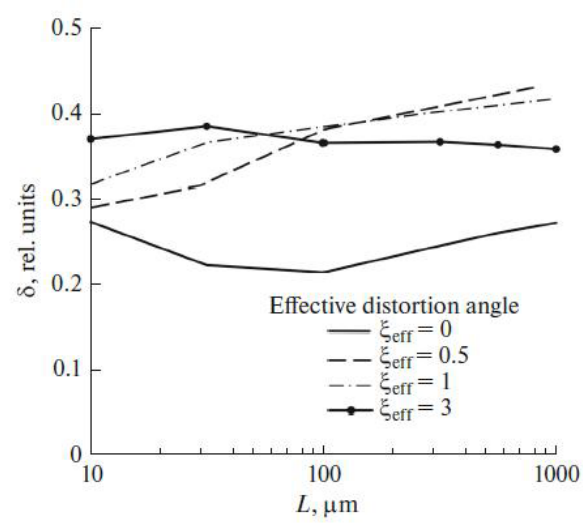

Figure 3. Linear depolarization ratio calculated for randomly oriented hexagonal columns with effective distortion angles of $0,0.5,1$, and 3 as a unction of the crystal length at a wavelength of $532 \mathrm{~nm}$

Thus, all the above written and Figs. 2 and 3 allow us to draw a conclusion that in $70 \%$ of $85 \%$ cases of observation of chaotically oriented particles in the period from December 2010, to February 2013, they had rather simple shapes of the hexagonal column type (bullet or bullet-rosette), and only in $15 \%$ of the cases the shape was of aggregate type.

\section{SUMMARY}

A moveable TRMPL lidar system is developed for cirrus detecting at Hefei, China. The depolarization ratios for cirrus cloud have been investigated from statistical characteristics. The parameters are matched with the developed model well and This allows us to retrieve, with some 
uncertainty, microphysical parameters of cirrus clouds.

\section{ACKNOWLEDGEMENTS}

This work was supported by the National Natural Science Foundation of China under Grant No. 41975038, 41590871, 41811530088 and the Youth Innovation Promotion Association CAS under Grant No. 2017482 and the CAS President's International Fellowship Initiative under Grant No. 2016VEA044.

\section{REFERENCES}

[1] Goldfarb, L., et al. J. Geophys. Res. Lett. 9(28), $1687-1690$ (2001).

[2]Heymsfield, A. J., et al. J. Atmos. Sci. 41, 846-855 (1984).

[3]Jensen, E. J., et al. J. Geophys. Res. 101, 2136121375 (1996).

[4]Chen, T., et al. J. Climate 13, 264-286 (2000).

[5]Nazaryan, H., et al. J. Geophys. Res. 113, D16211 (2008).

[6]Young, S. A., Appl. Opt. 34, 7019-7030 (1995).

[7]Wang, Z., et al. Optical Review 16, 566-570 (2009).

[8]Wang, Z., et al. Chinese Optics Letters 6, 235-237 (2008).

[9]Tao, Z., et al. Appl. Opt. 47, 1478-1485 (2008).

[10]Sassen, K., Bull. Am. Meteorol. Soc., 72, 18481866 (1991).

[11]Borovoi, A., et al. Optics Express 22(20), 2456624573 (2014).

[12]Sassen, K., et al. J. Atmos. Sci. 58(15), 2103-2112 (2001).

[13]Borovoi, A.,et al. J. Quant. Spectrosc. Radiat. Transfer. 146, 181-189 (2014).

[14]Yang, P., et al. Appl. Opt. 35(33), 6568-6584 (1996).

[15] A. Borovoi, et al. Opt. Lett. 39 (19), 5788-5791 (2014).

[16] A. Borovoi, et al. Opt. Express. 23 (19), 2455724571 (2015).

[17] D. L. Mitchell et al. J. Atmos. Sci. 51, 817-832 (1994). 